\title{
On indecomposability and composants of chaotic continua
}

\author{
by
}

\author{
Hisao Kato (Tsukuba)
}

\begin{abstract}
A homeomorphism $f: X \rightarrow X$ of a compactum $X$ with metric $d$ is expansive if there is $c>0$ such that if $x, y \in X$ and $x \neq y$, then there is an integer $n \in \mathbb{Z}$ such that $d\left(f^{n}(x), f^{n}(y)\right)>c$. A homeomorphism $f: X \rightarrow X$ is continuum-wise expansive if there is $c>0$ such that if $A$ is a nondegenerate subcontinuum of $X$, then there is an integer $n \in \mathbb{Z}$ such that $\operatorname{diam} f^{n}(A)>c$. Clearly, every expansive homeomorphism is continuum-wise expansive, but the converse assertion is not true. In [6], we defined the notion of chaotic continua of homeomorphisms and proved the existence of chaotic continua of continuum-wise expansive homeomorphisms. Also, we studied indecomposability of chaotic continua. In this paper, we investigate further more properties of indecomposability of chaotic continua and their composants. In particular, we prove that if $f: X \rightarrow X$ is a continuum-wise expansive homeomorphism of a plane compactum $X \subset \mathbb{R}^{2}$ with $\operatorname{dim} X>0$, then there exists a $\sigma$-chaotic continuum $Z$ ( $\sigma=\mathrm{s}$ or $\left.\mathrm{u}\right)$ of $f$ such that $Z$ is an indecomposable subcontinuum of $X$ and for each $z \in Z$ the composant $c(z)$ of $Z$ containing $z$ coincides with the continuum-wise $\sigma$-stable set $V^{\sigma}(z ; Z)$.
\end{abstract}

1. Introduction. All spaces considered in this paper are assumed to be separable metric spaces. Maps are continuous functions. By a compactum we mean a compact metric space. A continuum is a connected, nondegenerate compactum. A homeomorphism $f: X \rightarrow X$ of a compactum $X$ with metric $d$ is called expansive ([14] and [1]) if there is $c>0$ such that for any $x, y \in X$ and $x \neq y$, there is an integer $n \in \mathbb{Z}$ such that

$$
d\left(f^{n}(x), f^{n}(y)\right)>c .
$$

A homeomorphism $f: X \rightarrow X$ of a compactum $X$ is continuum-wise expansive (resp. positively continuum-wise expansive) [5] if there is $c>0$ such that if $A$ is a nondegenerate subcontinuum of $X$, then there is an integer

1991 Mathematics Subject Classification: Primary 54H20, 54F50; Secondary 54E50, $54 \mathrm{~B} 20$.

Key words and phrases: expansive homeomorphism, continuum-wise expansive homeomorphism, indecomposable, composant, chaotic continuum, plane compactum, stable and unstable sets. 
$n \in \mathbb{Z}$ (resp. a natural number $n \geq 0$ ) such that

$$
\operatorname{diam} f^{n}(A)>c,
$$

where $\operatorname{diam} B=\sup \{d(x, y) \mid x, y \in B\}$ for a set $B$. Such a positive number $c$ is called an expansive constant for $f$. Note that each expansive homeomorphism is continuum-wise expansive, but the converse assertion is not true. There are many continuum-wise expansive homeomorphisms which are not expansive (e.g., see [5]). By the definitions, we see that expansiveness and continuum-wise expansiveness do not depend on the choice of the metric $d$ of $X$. These notions have been extensively studied in topological dynamics, ergodic theory and continuum theory.

Let $X$ be a compactum. The hyperspace $2^{X}$ of $X$ is the set of all nonempty closed subsets of $X$ with the Hausdorff metric $d_{\mathrm{H}}$. Let

$$
C(X)=\left\{A \in 2^{X} \mid A \text { is connected }\right\} .
$$

Note that $2^{X}$ and $C(X)$ are compacta (e.g., see [8] or [10]).

A continuum $X$ is decomposable if there are two proper subcontinua $A$ and $B$ of $X$ such that $A \cup B=X$. A continuum $X$ is indecomposable if it is not decomposable. Let $X$ be a continuum and let $p \in X$. Then the set

$c(p)=\{x \in X \mid$ there is a proper subcontinuum $A$ of $X$ containing $p$ and $x\}$ is called the composant of $X$ containing $p$. See [8] for some fundamental properties of indecomposable continua and composants.

For a homeomorphism $f: X \rightarrow X$, we define sets of stable and unstable nondegenerate subcontinua of $X$ as follows (see [6]):

$$
\begin{array}{r}
\mathbb{V}^{\mathrm{s}}\left(=\mathbb{V}_{f}^{\mathrm{s}}\right)=\{A \mid A \text { is a nondegenerate subcontinuum of } X \text { such that } \\
\left.\lim _{n \rightarrow \infty} \operatorname{diam} f^{n}(A)=0\right\}, \\
\mathbb{V}^{\mathrm{u}}\left(=\mathbb{V}_{f}^{\mathrm{u}}\right)=\{A \mid A \text { is a nondegenerate subcontinuum of } X \text { such that } \\
\left.\lim _{n \rightarrow \infty} \operatorname{diam} f^{-n}(A)=0\right\} .
\end{array}
$$

For each $0<\delta<\varepsilon$, put

$$
\begin{aligned}
\mathbb{V}^{\mathrm{s}}(\delta ; \varepsilon) & =\left\{A \in C(X) \mid \operatorname{diam} A \geq \delta, \text { and } \operatorname{diam} f^{n}(A) \leq \varepsilon \text { for each } n \geq 0\right\} \\
\mathbb{V}^{\mathrm{u}}(\delta ; \varepsilon) & =\left\{A \in C(X) \mid \operatorname{diam} A \geq \delta, \text { and } \operatorname{diam} f^{-n}(A) \leq \varepsilon \text { for each } n \geq 0\right\} .
\end{aligned}
$$

Similarly, for each closed subset $Z$ of $X$ and $x \in Z$, the continuum-wise $\sigma$-stable sets $V^{\sigma}(x ; Z)$ of $f$ are defined as follows:

$V^{\mathrm{s}}(x ; Z)=\{y \in Z \mid$ there is $A \in C(Z)$ such that $x, y \in A$

$$
\text { and } \left.\lim _{n \rightarrow \infty} \operatorname{diam} f^{n}(A)=0\right\},
$$

$V^{\mathrm{u}}(x ; Z)=\{y \in Z \mid$ there is $A \in C(Z)$ such that $x, y \in A$

$$
\text { and } \left.\lim _{n \rightarrow \infty} \operatorname{diam} f^{-n}(A)=0\right\} \text {. }
$$


Let $f: X \rightarrow X$ be a homeomorphism of a compactum $X$ with $\operatorname{dim} X>0$. Then a subcontinuum $Z$ of $X$ is called a $\sigma$-chaotic continuum of $f$ (where $\sigma=\mathrm{s}, \mathrm{u})$ if

(1) for each $x \in Z, V^{\sigma}(x ; Z)$ is dense in $Z$, and

(2) there is $\tau>0$ such that for each $x \in Z$ and each neighborhood $U$ of $x$ in $X$, there is $y \in U \cap Z$ such that

$$
\begin{aligned}
\liminf _{n \rightarrow \infty} d\left(f^{n}(x), f^{n}(y)\right) \geq \tau & \text { in case } \sigma=\mathrm{s}, \\
\liminf _{n \rightarrow \infty} d\left(f^{-n}(x), f^{-n}(y)\right) \geq \tau & \text { in case } \sigma=\mathrm{u} .
\end{aligned}
$$

Note that $\mathbb{V}^{\sigma}(\delta ; \varepsilon)(\sigma=\mathrm{u}, \mathrm{s})$ is closed in $C(X)$. Also, note that if $f$ : $X \rightarrow X$ is a continuum-wise expansive homeomorphism with an expansive constant $c>0$, then (1) for each $0<\delta<\varepsilon<c, \mathbb{V}^{\sigma}(\delta ; \varepsilon) \subset \mathbb{V}^{\sigma}$, and $\mathbb{V}^{\sigma}$ is an $F_{\sigma}$-set in $C(X)$, and $(2) V^{\mathrm{u}}(z ; Z)$ is a connected $F_{\sigma}$-set containing $z$, because $V^{\mathrm{u}}(z ; Z)=\bigcup_{n=0}^{\infty}\left(\bigcup\left\{A \in C(Z) \mid z \in A, \operatorname{diam} f^{-i}(A) \leq \varepsilon\right.\right.$ for $\left.i \geq n\right\}$ ) (see $[5,(2.1)])$. Similarly, $V^{\mathrm{s}}(z ; Z)$ is a connected $F_{\sigma}$-set containing $z$.

In [6], we showed that if $f: X \rightarrow X$ is a continuum-wise expansive homeomorphism of a compactum $X$ with $\operatorname{dim} X>0$, then there exists a chaotic continuum of $f$ (see $[6,(3.6)]$ ). In this case, if $Z$ is a $\sigma$-chaotic continuum of $f$, then the decomposition $\left\{V^{\sigma}(z ; Z) \mid z \in Z\right\}$ of $Z$ is an uncountable family of mutually disjoint, connected $F_{\sigma}$-sets.

2. Indecomposability of chaotic continua and their composants. In [6], we studied indecomposability of chaotic continua of continuum-wise expansive homeomorphisms. In this section, we investigate further properties of indecomposability of chaotic continua and their composants. We need the following results.

Lemma (2.1) (see the proof of $[5,(2.3)]$ ). Let $f: X \rightarrow X$ be a continuumwise expansive homeomorphism of a compactum $X$ with an expansive constant $c>0$, and let $0<\varepsilon<c / 2$. Then there is $\varepsilon>\delta>0$ such that if $A$ is a subcontinuum of $X$ with $\operatorname{diam} A \leq \delta$ and $\operatorname{diam} f^{m}(A) \geq \varepsilon$ for some $m \in \mathbb{Z}$, then one of the following two conditions holds:

1. If $m \geq 0$, then for each $n \geq m$ and $x \in f^{n}(A)$, there is a subcontinuum $B$ of $A$ such that $x \in f^{n}(B), \operatorname{diam} f^{j}(B) \leq \varepsilon$ for $0 \leq j \leq n$ and $\operatorname{diam} f^{n}(B)=\delta$.

2. If $m<0$, then for each $n \geq-m$ and $x \in f^{-n}(A)$, there is a subcontinuum $B$ of $A$ such that $x \in f^{-n}(B)$, diam $f^{-j}(B) \leq \varepsilon$ for $0 \leq j \leq n$, and $\operatorname{diam} f^{-n}(B)=\delta$.

Lemma (2.2) ([5, (2.4)]). Let $f: X \rightarrow X$ be a continuum-wise expansive homeomorphism of a compactum $X$ with $\operatorname{dim} X>0$. Then:

1. $\mathbb{V}^{\mathrm{u}} \neq \emptyset$ or $\mathbb{V}^{\mathrm{s}} \neq \emptyset$. 
2. If $\delta>0$ is as in the above lemma, then for each $\gamma>0$ there is a natural number $N(\gamma)$ such that if $A$ is a subcontinuum of $X$ with $\operatorname{diam} A \geq \gamma$, then either $\operatorname{diam} f^{n}(A) \geq \delta$ for each $n \geq N(\gamma)$ or $\operatorname{diam} f^{-n}(A) \geq \delta$ for each $n \geq N(\gamma)$.

Lemma (2.3) ([4, (1.5) and (1.1)]). Let $f: X \rightarrow X$ be a continuumwise expansive homeomorphism of a compactum $X$ with $\operatorname{dim} X>0$ and let $c>0$ be an expansive constant for $f$. Then $f$ is positively continuum-wise expansive if and only if for any $c>\varepsilon>0$ there is a positive number $\delta>0$ such that if $A \in C(X)$ with $\operatorname{diam} A \leq \delta$, then $\operatorname{diam} f^{-n}(A) \leq \varepsilon$ for any $n \geq 0$, and hence $A \in \mathbb{V}^{\mathrm{u}}$.

Note that for a continuum-wise expansive homeomorphism $f: X \rightarrow X$, $f$ is positively continuum-wise expansive if and only if $\mathbb{V}^{s}=\emptyset$. First, we shall prove the following theorem.

Theorem (2.4). Suppose that $f: X \rightarrow X$ is a positively continuum-wise expansive homeomorphism of a compactum $X$ with $\operatorname{dim} X>0$. Then:

(1) There is a $\mathrm{u}$-chaotic continuum $Z$ of $f$ such that $Z$ is indecomposable, and for each $z \in Z$ the composant $c(z)$ of $Z$ containing the point $z$ coincides with the continuum-wise $\mathrm{u}$-stable set $V^{\mathrm{u}}(z ; Z)$ of $Z$.

(2) $Z$ is an indecomposable subcontinuum of $X$ such that for each $z \in Z$, the composant $c(z)$ of $Z$ containing $z$ coincides with $V^{\mathrm{u}}(z ; Z)$ if and only if $Z$ is a minimal element of the set of all $\mathrm{u}$-chaotic continua of $f$.

Proof. Let $c>0$ be an expansive constant for $f$ and let $0<\varepsilon<c / 2$. Choose $\delta>0$ satisfying the conditions of (2.1) and (2.3). By $(2.3), \mathbb{V}^{\mathrm{u}}(\delta ; \varepsilon)$ $\neq \emptyset$. Since $f$ is positively continuum-wise expansive, $\mathbb{V}^{\mathrm{s}}(\delta ; \varepsilon)=\emptyset$.

For each pair $(y ; Y)$ with $y \in Y \in C(X)$, consider the following condition (see the proof of $[6,(3.6)])$ :

$P(y ; Y) \quad \mathrm{Cl}\left(V^{\mathrm{u}}(y ; Y)\right)=Y$, and for each $n \geq 0$, there is $A_{n} \in V^{\mathrm{u}}(\delta ; \varepsilon)$ such that $f^{-n}(y) \in A_{n}$ and $f^{n}\left(A_{n}\right) \subset V^{\mathrm{u}}(y ; Y)$, i.e., $y \in f^{n}\left(A_{n}\right)$ $\subset Y$.

Also, define

$$
\Gamma=\{Y \in C(X) \mid \text { there is } y \in Y \text { such that } P(y ; Y) \text { holds }\} .
$$

By the proof of $[6,(3.6)]$, there is a minimal element of $\Gamma$. If $Z$ is a minimal element of $\Gamma$, then $Z$ is a u-chaotic continuum of $f$. We shall show that for each $z \in Z$ the composant $c(z)$ of $Z$ containing $z$ coincides with $V^{\mathrm{u}}(z ; Z)$. Let $B$ be a proper subcontinuum of $Z$. Choose $b \in B$. Suppose, on the contrary, that $B \notin \mathbb{V}^{\mathrm{u}}$. Then by (2.3) we see that $\operatorname{diam} f^{-n}(B)>\delta$ for each $n \geq 0$. Let $B^{\prime}=\mathrm{Cl}\left(V^{\mathrm{u}}(b ; B)\right)$. By $(2.3), P\left(b ; B^{\prime}\right)$ holds. Hence $B^{\prime} \in \Gamma$. Since $Z$ is a minimal element of $\Gamma, Z=B^{\prime} \subset B$. This is a contradiction. Hence $B \subset V^{\mathrm{u}}(b ; Z)$, which implies that for each $z \in Z$, 
$c(z)=V^{\mathrm{u}}(z ; Z)$. Since the family $\left\{V^{\mathrm{u}}(z ; Z)(=c(z)) \mid z \in Z\right\}$ is a family of uncountable disjoint $F_{\sigma}$-sets, we see that $Z$ is indecomposable.

Next, we shall show (2). If a subcontinuum $Z$ is indecomposable and $c(z)=V^{\mathrm{u}}(z ; Z)$ for each $z \in Z$, we see that $Z$ is a weakly u-chaotic continuum of $f$ (see [6]) and by $[6,(3.7)], Z$ is a u-chaotic continuum of $f$. Since $c(z)=V^{\mathrm{u}}(z ; Z)$ for each $z \in Z$, we see that $Z$ is a minimal u-chaotic continuum of $f$. We shall show the converse assertion. If $Z$ is a u-chaotic continuum of $f$, then $Z \in \Gamma$. Hence $Z$ is a minimal element of $\Gamma$ if and only if $Z$ is a minimal u-chaotic continuum of $f$. Using this fact and the proof of (1), we see that if $Z$ is a minimal u-chaotic continuum of $f$, then $Z$ is indecomposable and for each $z \in Z$ the composant $c(z)$ of $Z$ containing $z$ coincides with $V^{\mathrm{u}}(z ; Z)$.

A homeomorphism $f: X \rightarrow X$ of a continuum $X$ is continuum-wise fully expansive (resp. positively continuum-wise fully expansive) [4] provided that for any $\varepsilon>0$ and $\delta>0$, there is a natural number $N=N(\varepsilon ; \delta)>0$ such that if $A \in C(X)$ and $\operatorname{diam} A \geq \delta$, then either $d_{\mathrm{H}}\left(f^{n}(A), X\right)<\varepsilon$ for all $n \geq N$, or $d_{\mathrm{H}}\left(f^{-n}(A), X\right)<\varepsilon$ for all $n \geq N$ (resp. $d_{\mathrm{H}}\left(f^{n}(A), X\right)<\varepsilon$ for all $n \geq N)$.

Corollary (2.5). If $f: X \rightarrow X$ is a positively continuum-wise fully expansive homeomorphism of a continuum $X$, then $X$ is the only $\mathrm{u}$-chaotic continuum of $f$, it is indecomposable and for each $x \in X$ the composant $c(x)$ of $X$ containing $x$ coincides with $V^{\mathrm{u}}(x ; X)$.

Pro of. The proof is similar to the above. By $[6,(3.15)], X$ is a u-chaotic continuum of $f$. Since $f$ is positively expansive, we can choose $\delta>0$ as in (2.1) and (2.3). Let $B$ be any proper subcontinuum of $X$. We shall show that $B \in \mathbb{V}^{\mathrm{u}}$. Suppose, on the contrary, that diam $f^{-n}(B)>\delta$ for each $n \geq 0$. Since $B=f^{n}\left(f^{-n}(B)\right)$ and $\operatorname{diam} f^{-n}(B)>\delta$ for all $n \geq 0, d_{\mathrm{H}}(B, X)=0$ and hence $X=B$, because $f$ is a positively continuum-wise fully expansive homeomorphism. This implies that for each $x \in X, c(x)=V^{\mathrm{u}}(x ; X)$. Also, we see that $X$ is indecomposable.

Let $f: X \rightarrow X$ be a map of a compactum $X$. Consider the following sets:

(1) $\mathcal{I}(f)=\left\{A \in 2^{X} \mid A\right.$ is $f$-invariant $\}$.

(2) $\mathcal{I}^{+}(f)=\{A \in \mathcal{I}(f) \mid \operatorname{dim} A>0\}$.

(3) $\mathcal{M}^{+}(f)=\left\{A \in \mathcal{I}^{+}(f) \mid\right.$ if $B \in \mathcal{I}(f)$ and $B$ is a proper subset of $A$, then $B$ is 0 -dimensional $\}$, i.e., $\mathcal{M}^{+}(f)$ is the set of all minimal elements of $\mathcal{I}^{+}(f)$.

The following theorem is the main result of this paper. 
TheOrem (2.6). Let $X$ be a compactum in the plane $\mathbb{R}^{2}$ with $\operatorname{dim} X>0$. If $f: X \rightarrow X$ is a continuum-wise expansive homeomorphism, then for some $\sigma=\mathrm{u}$ or $\mathrm{s}$ there is a $\sigma$-chaotic continuum $Z$ of $f$ such that $Z$ is indecomposable, and for each $z \in Z$ the composant $c(z)$ of $Z$ containing $z$ coincides with $V^{\sigma}(z ; Z)$. In particular, $X$ contains an indecomposable chaotic continuum of $f$.

Proof. By $[7,(3.1)], \mathcal{M}^{+}(f) \neq \emptyset$. Choose $Y \in \mathcal{M}^{+}(f)$. Without loss of generality, we may assume that $X=Y$, i.e., $X \in \mathcal{M}^{+}(f)$. Also, by (2.2) we may assume that $\mathbb{V}^{\mathrm{u}} \neq \emptyset$. First, we shall prove that $\mathbb{V}^{\mathrm{s}}=\emptyset$. Suppose, on the contrary, that $\mathbb{V}^{\mathrm{s}} \neq \emptyset$. Choose $A \in \mathbb{V}^{\mathrm{s}}$. Note that $\mathrm{Cl}\left(\bigcup_{i=0}^{\infty} f^{-i}(A)\right)=X$ $(=Y)$, because $X \in \mathcal{M}^{+}(f)$ (see the proof of [7, (3.1)]). By using this fact and (2.1), we see that for any $x \in X$, there is a subcontinum $A_{x} \in \mathbb{V}^{\mathrm{s}}(\delta ; \varepsilon)$ with $x \in A_{x}$, where $\delta, \varepsilon$ are positive numbers as in (2.1). Similarly, we see that for each $x \in X$, there are two subcontinua $A_{x}, B_{x}$ of $X$ such that $A_{x} \in \mathbb{V}^{\mathrm{s}}(\delta ; \varepsilon), B_{x} \in \mathbb{V}^{\mathrm{u}}(\delta ; \varepsilon)$ and $x \in A_{x} \cap B_{x}$.

By [3] and [5, (6.2)], $X$ is not locally connected. Hence we can choose a disk $D=\langle a, b, c, d\rangle$ in $\mathbb{R}^{2}$ (see Figure 1 ) such that there are components $C_{n}$ $(n=1,2, \ldots)$ of $D \cap X$ satisfying $C_{n} \cap[a, c] \neq \emptyset \neq C_{n} \cap[b, d], \lim _{n \rightarrow \infty} C_{n}=K$ is contained in $C$, where $C$ is a component of $D \cap X$ (see [8, p. 245]). We may assume that there is a sequence $A_{1}, A_{2}, \ldots$ of elements of $V^{\mathrm{s}}(\delta ; \varepsilon)$ such that $A_{n} \subset C_{n}, A_{n} \cap[a, c] \neq \emptyset \neq A_{n} \cap[b, d]$. Choose a sequence $B_{1}, B_{2}, \ldots$ of elements of $\mathbb{V}^{\mathrm{u}}(\gamma ; \varepsilon)$ such that $B_{n} \subset C_{n}$, where $\gamma$ is some positive number. Note that $A_{i} \cap B_{j}=\emptyset$ for $i \neq j$. We may assume that $\lim _{n \rightarrow \infty} A_{n}=A_{0} \in$ $\mathbb{V}^{\mathrm{s}}(\delta ; \varepsilon)$ and $\lim _{n \rightarrow \infty} B_{n}=B_{0} \in \mathbb{V}^{\mathrm{u}}(\gamma ; \varepsilon)$. Then $B_{0} \subset A_{0}$. Then we see that $\operatorname{diam} f^{n}\left(B_{0}\right) \leq \varepsilon$ for each $n \in \mathbb{Z}$ (see the proof of [4, (4.10)]). This is a contradiction. Therefore $V^{\mathrm{s}}=\emptyset$, which implies that $f$ is positively continuum-wise expansive. Consequently, we see that if $X \in \mathcal{M}^{+}(f)$ and $X$ is a plane compactum, then either $f: X \rightarrow X$ or $f^{-1}: X \rightarrow X$ is positively continuum-wise expansive. (2.4) implies that there is a desired chaotic continuum $Z$ in $X$. This completes the proof.

Let $\mathcal{P}$ be a family of compact polyhedra. A continuum $X$ is called a $\mathcal{P}$-like continuum if for any $\varepsilon>0$ there is an onto map $g: X \rightarrow P$ such that $P \in \mathcal{P}$ and $\operatorname{diam} g^{-1}(y)<\varepsilon$ for each $y \in P$.

Corollary (2.7). Let $\mathbf{F}$ be a finite family of graphs and let $X$ be an $\mathbf{F}$-like continuum. If $f: X \rightarrow X$ is a continuum-wise expansive homeomorphism, then there is a $\sigma$-chaotic continuum $Z$ of $f$ such that $Z$ is indecomposable, and for each $z \in Z$ the composant $c(z)$ of $Z$ containing $z$ coincides with $V^{\sigma}(z ; Z)$.

Proof. Since $\mathcal{M}^{+}(f) \neq \emptyset$, we can choose $Y \in \mathcal{M}^{+}(f)$. Let $f^{\prime}=f \mid Y$ : $Y \rightarrow Y$. We shall show that either $f^{\prime}$ or $f^{\prime-1}$ is positively continuum-wise 


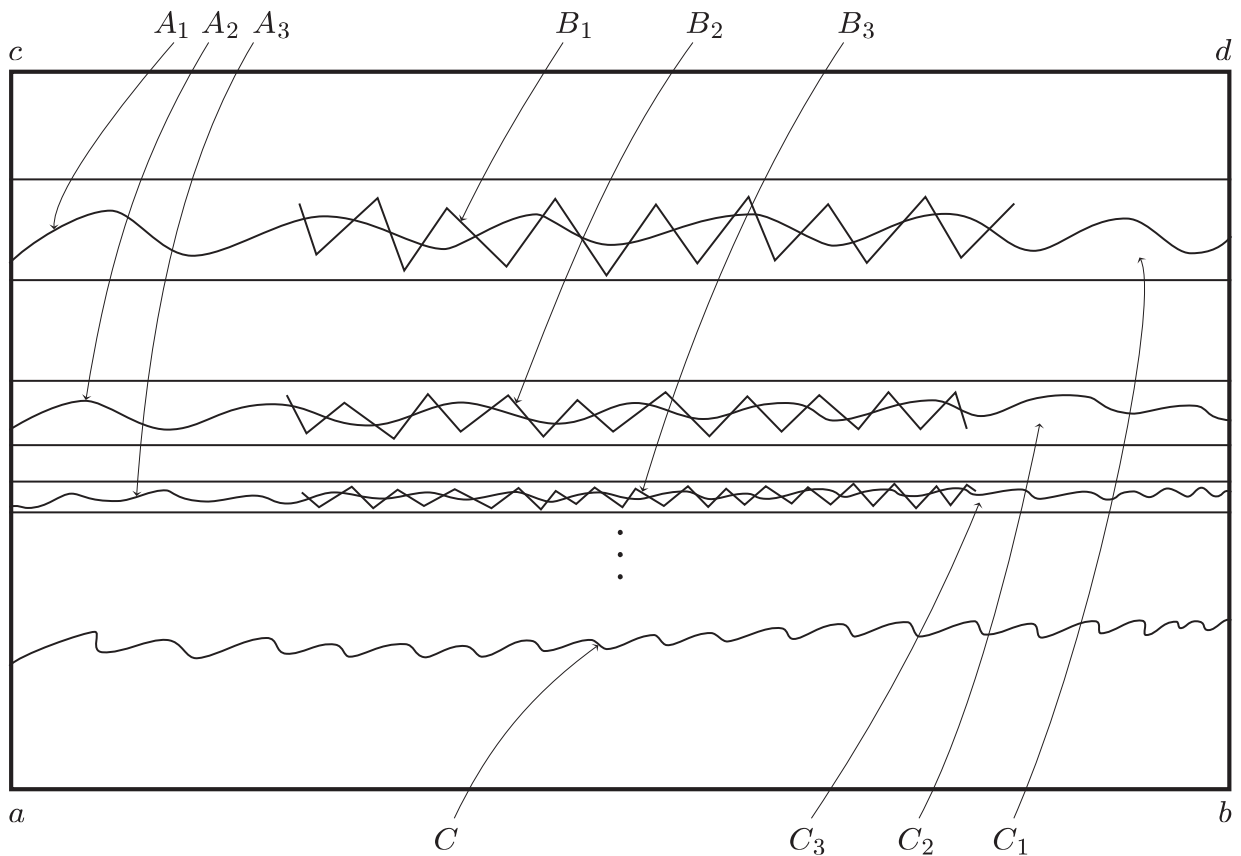

Fig. 1

expansive. Suppose, on the contrary, that $\mathbb{V}_{f^{\prime}}^{\mathrm{u}} \neq \emptyset \neq \mathbb{V}_{f^{\prime}}^{\mathrm{s}}$. Then we see that for each $y \in Y$, there are $A_{y} \in \mathbb{V}_{f^{\prime}}^{\mathrm{u}}$ and $B_{y} \in \mathbb{V}_{f^{\prime}}^{\mathrm{s}}$ such that $y \in A_{y} \cap B_{y}$. Note that $A_{y}-B_{y} \neq \emptyset \neq B_{y}-A_{y}$, because $f^{\prime}$ is continuum-wise expansive and hence $\operatorname{dim}\left(A_{y} \cap B_{y}\right)=0$. By using this fact, for each $n \geq 1$ we can easily prove the following condition:

$(*)_{n} \quad$ There is a subcontinuum $A \in \mathbb{V}_{f^{\prime}}^{\mathrm{u}}$ and $n$ subcontinua $B_{1}, \ldots, B_{n} \in$ $\mathbb{V}_{f^{\prime}}^{\mathrm{s}}$ such that $A \cap B_{i} \neq \emptyset$ and $B_{i} \cap B_{j}=\emptyset(i \neq j)$.

Note that $B_{i}-A \neq \emptyset$ for each $i$. Since $X$ is $\mathbf{F}$-like, there is an onto map $g: X \rightarrow G \in \mathbf{F}$ such that $g(A) \cap g\left(B_{i}\right) \neq \emptyset, g\left(B_{i}\right)-g(A) \neq \emptyset$ for each $i=1, \ldots, n$ and $g\left(B_{i}\right) \cap g\left(B_{j}\right)=\emptyset(i \neq j)$. Note that $\mathbf{F}$ is a finite family of graphs. This is a contradiction, because $(*)_{n}$ holds for each $n$. Hence either $f^{\prime}$ or $f^{\prime-1}$ is positively continuum-wise expansive. By (2.4), we obtain the desired chaotic continuum $Z$ of $f$.

A map $f: X \rightarrow X$ of a compactum $X$ has sensitive dependence on initial conditions if there is $c>0$ such that for each $x \in X$ and each neighborhood $U$ of $x$ in $X$ there is a point $y \in U$ such that $d\left(f^{n}(x), f^{n}(y)\right) \geq c$ for some $n \geq 0$. For any map $f: X \rightarrow X$ of a compactum $X$, consider the inverse limit space $(X, f)$ and the map $\widetilde{f}:(X, f) \rightarrow(X, f)$ defined by

$$
(X, f)=\left\{\left(x_{n}\right)_{n=0}^{\infty} \mid x_{n} \in X, f\left(x_{n+1}\right)=x_{n} \text { for each } n\right\},
$$


and

$$
\widetilde{f}\left(x_{0}, x_{1}, \ldots,\right)=\left(f\left(x_{0}\right), x_{0}, \ldots\right) .
$$

Then $(X, f)$ is a compactum and the homeomorphism $\tilde{f}:(X, f) \rightarrow(X, f)$ is called the shift map of $f$. By [7, (5.4)] and (2.5), we obtain the following.

Corollary (2.8). Suppose that a map $f: G \rightarrow G$ of a finite graph $G$ has sensitive dependence on initial conditions and $\widetilde{f}:(G, f) \rightarrow(G, f)$ is the shift map of $f$. Then $\mathbf{M}^{+}(\tilde{f})=\left\{Y_{1}, \ldots, Y_{N}\right\}$ is nonempty and a finite set and the following conditions are satisfied:

(1) $Y_{i} \cap Y_{j}(i \neq j)$ is empty or a finite set of periodic points of $\tilde{f}$.

(2) Both $\widetilde{f}$ and $\widetilde{f}^{-1}$ are chaotic on each $Y_{i}$ in the sense of Devaney, and there is a continum $Z_{i}$ in $Y_{i}$ and a natural number $n(i) \geq 1$ such that $Z_{i}$ is $\widetilde{f}^{n(i)}$-invariant, $\bigcup_{k=0}^{n(i)-1} \widetilde{f}^{k}\left(Z_{i}\right)=Y_{i}, \widetilde{f}^{k}\left(Z_{i}\right) \cap \widetilde{f}^{k^{\prime}}\left(Z_{i}\right)(0 \leq k \neq$ $\left.k^{\prime} \leq n(i)-1\right)$ is empty or a finite set of periodic points of $\tilde{f}$ for each $i$, and $\tilde{f}^{n(i)} \mid \widetilde{f}^{k}\left(Z_{i}\right)$ is topologically mixing and hence positively continuum-wise fully expansive. In particular, $Z_{i}$ is a u-chaotic continuum of $\tilde{f}$ such that $Z_{i}$ is an indecomposable continuum and for each $z \in Z_{i}$, the composant $c(z)$ of $Z_{i}$ containing $z$ coincides with $V^{\mathrm{u}}\left(z ; Z_{i}\right)$.

(3) There is an $\tilde{f}$-invariant closed subset $F(\tilde{f})$ in $\mathrm{Cl}\left((G, f)-\bigcup_{i=1}^{N} Y_{i}\right)$ such that $\operatorname{dim} F(\widetilde{f}) \leq 0$, and if $x \in\left[(G, f)-\left(\bigcup_{i=1}^{N} Y_{i} \cup F(\widetilde{f})\right)\right]$ and any $\varepsilon>0$, there is a neighborhood $U$ of $x$ in $(G, f)$ and a natural number $n(\varepsilon) \geq 1$ such that $\tilde{f}^{n}(U)$ is contained in the $\varepsilon$-neighborhood of $\bigcup_{i=1}^{N} Y_{i}$ in $(G, f)$ for each $n \geq n(\varepsilon)$.

3. Examples. In this section, we give some examples which are related to results obtained in the previous section.

ExAmple (3.1). Let $S^{1}$ be the unit circle and $f: S^{1} \rightarrow S^{1}$ be the natural covering map with degree 2. Consider the inverse limit space $X=\left(S^{1}, f\right)$ and the shift map $\widetilde{f}:\left(S^{1}, f\right) \rightarrow\left(S^{1}, f\right)$ of $f$. Then $g=\widetilde{f}: X \rightarrow X$ is (positively continuum-wise fully) expansive. Note that $g \times g: X \times X \rightarrow$ $X \times X$ is a positively continuum-wise expansive homeomorphism. Moreover, $X \times X$ is itself a u-chaotic continuum of $g \times g$, but it is decomposable. For each $x \in X, X \times\{x\}$ and $\{x\} \times X$ are minimal u-chaotic continua of $g \times g$ which are indecomposable.

ExAmple (3.2). Let $f: T^{2} \rightarrow T^{2}$ be an Anosov diffeomorphism, say $\left[\begin{array}{ll}2 & 1 \\ 1 & 1\end{array}\right]$ on the 2-dimensional torus $T^{2}=S^{1} \times S^{1}$. Then $f: T^{2} \rightarrow T^{2}$ is (continuumwise fully) expansive, but neither $f$ nor $f^{-1}$ are positively continuum-wise expansive. Note that $T^{2}$ is a $\sigma$-chaotic continuum of $f$ for each $\sigma=u$, s. Since $f$ is continuum-wise fully expansive, we see that $T^{2}$ is the only chaotic continuum of $f$ (see the proof of $(2.5)$ ). In this case, there is no chaotic 
continuum $Z$ of $f$ which is indecomposable. Hence in the statement of (2.6), we cannot omit the condition that $X$ is a plane compactum.

\section{References}

[1] N. Aoki, Topological dynamics, in: Topics in General Topology, K. Morita and J. Nagata (eds.), Elsevier, 1989, 625-740.

[2] J. F. Jacobson and W. R. Utz, The nonexistence of expansive homeomorphisms of a closed 2-cell, Pacific J. Math. 10 (1960), 1319-1321.

[3] H. Kato, The nonexistence of expansive homeomorphisms of Peano continua in the plane, Topology Appl. 34 (1990), 161-165.

[4] -, Concerning continuum-wise fully expansive homeomorphisms of continua, ibid. 53 (1993), 239-258.

[5] -, Continuum-wise expansive homeomorphisms, Canad. J. Math. 45 (1993), 576598.

[6] -, Chaotic continua of (continuum-wise) expansive homeomorphisms and chaos in the sense of Li and Yorke, Fund. Math. 145 (1994), 261-279.

[7] -, Chaos of continuum-wise expansive homeomorphisms and dynamical properties of sensitive maps of graphs, Pacific J. Math., to appear.

[8] K. Kuratowski, Topology, Vol. II, Academic Press, New York, 1968.

[9] R. Mañé, Expansive homeomorphisms and topological dimension, Trans. Amer. Math. Soc. 252 (1979), 313-319.

[10] S. B. Nadler, Jr., Hyperspaces of Sets, Pure and Appl. Math. 49, Dekker, New York, 1978.

[11] T. O'Brien and W. Reddy, Each compact orientable surface of positive genus admits an expansive homeomorphism, Pacific J. Math. 35 (1970), 737-741.

[12] R. V. Plykin, On the geometry of hyperbolic attractors of smooth cascades, Russian Math. Surveys 39 (1984), 85-131.

[13] W. Reddy, The existence of expansive homeomorphisms of manifolds, Duke Math. J. 32 (1965), 627-632.

[14] W. Utz, Unstable homeomorphisms, Proc. Amer. Math. Soc. 1 (1950), 769-774.

[15] R. F. Williams, A note on unstable homeomorphisms, ibid. 6 (1955), 308-309.

Institute of Mathematics

University of Tsukuba

Ibaraki 305, Japan

E-mail: hisakato@sakura.cc.tsukuba.ac.jp 